\title{
Selective Synthesis Combined with Chemical Separation of Single-Walled Carbon Nanotubes for Chirality Selection
}

Xiaolin Li, Xiaomin Tu, Sasa Zaric, Kevin Welsher, Won Seok Seo, Wei Zhao, Hongjie Dai*

Department of Chemistry and Laboratory for Advanced Materials, Stanford University, Stanford, CA 94305, USA

Department of Chemistry, University of Arkansas, Little Rock, AR 72204, USA

\section{Supplementary Information with Supplementary Figures}

\section{(1) Growth, purification and the dispersion of FeRu tubes}

The FeRu tube synthesis was preformed on a silica support Fe-Ru bimetallic catalyst using $\mathrm{CH}_{4} \mathrm{CVD}$ growth. The FeRu catalyst was prepared by sonicating a mixture of silica support (1g, Degussa Aerosil with $7 \mathrm{~nm}$ average particle size and BET surface area of $\left.380 \pm 30 \mathrm{~m}^{2} / \mathrm{g}\right)$ and iron nitrate $(0.36 \mathrm{mmol}, 145.44 \mathrm{mg})$ and ruthenium chloride hydrate $(0.36 \mathrm{mmol}, 74.67 \mathrm{mg})$ in methanol for $1 \mathrm{~h}$. Solid catalysts were obtained after removal of the solvent by rotary evaporator. SWNTs were grown by first flushing a 1" CVD tube furnace with $200 \mathrm{sccm}$ (standard cubic centimeter per minute) $\mathrm{H}_{2}$ for $10 \mathrm{mins}$, heating up to the growth temperature in $200 \mathrm{sccm}$ of $\mathrm{H}_{2}$ and then maintaining at the growth temperature in $200 \mathrm{sccm} \mathrm{CH} 4$ for 8 mins. The system was then cooled in $200 \mathrm{sccm}$ of $\mathrm{H}_{2}$. The SWNTs grown by this method were mixed with the silica support and FeRu 
nanoparticles. To remove the silica from the mixture, the solid product was etched in $15 \%$ HF solution in water $(75 \%)$ and ethanol (10\%) overnight at room temperature. The solid product was then washed thoroughly with ethanol and water to neutral $\mathrm{pH}$ and stored for further use including characterizations and chromatography separation. For characterizations, the nanotubes were added to an aqueous solution of a sodium cholate hydrate $(2 \% \mathrm{w} / \mathrm{v})$ surfactant and bath-sonicated $(17 \mathrm{~W}$ output) for $1 \mathrm{~h}$ to form a homogeneous suspension. The suspension was centrifuged at $24,000 \mathrm{~g}$ for $6 \mathrm{~h}$ to remove metallic catalyst particles and large bundles. The supernatant contained mostly individual SWNTs well suspended in water with the surfactant molecules adsorbed.

\section{(2) Ion exchange chromatography separation of $850^{\circ} \mathrm{C}$ growth $\mathrm{FeRu}$ tubes}

SWNT dispersion by single-strand DNA (ssDNA) and IEC (ion-exchange chromatography) separation were conducted according to the procedures described previously $^{1,2,3}$, with some changes outlined below. For ion exchange chromatography (IEC) separation, the FeRu grown SWNTs product after silica-support etching $(1 \mathrm{mg} / \mathrm{mL})$ was dispersed with $\mathrm{d}(\mathrm{GT})_{20}(1 \mathrm{mg} / \mathrm{mL})$ in $0.1 \mathrm{M} \mathrm{NaCl}$ solution through $2 \mathrm{hr}$ sonication. The suspension was then centrifuged at $16,000 \mathrm{~g}$ to get mostly individual SWNTs in the

\footnotetext{
${ }^{1}$ Huang, X.; Mclean, R. S.; Zheng, M. Anal. Chem. 2005, 77, 6225-6228.

2 Zheng, M.; Jagota, A.; Semke, E. D.; Diner, B. A.; Mclean, R. S.; Lustig, S. R.; Richardson, R. E.; Tassi, N. G. Nature Mater. 2003, 2, 338-342.

${ }^{3}$ Zheng, M.; Jagota, A.; Strano, M. S.; Santos, A. P.; Barone, P.; Chou, S. G.; Diner, B. A.; Dresselhaus, M. S.; Mclean, R. S.; Onoa, G. B.; Samsonidze, G. G.; Semke, E. D.; Usrey, M.; Walls, D. J. Science 2003, 302, 1545-1548.
} 
supernatant. A volume of $600 \mu \mathrm{L}$ of the suspension was injected into an anion-exchange column NS 1500 (75 mm by $7.8 \mathrm{~mm}$, Biochrom Labs, Inc., Terre Haute, IN) mounted on the Waters Breeze HPLC system. The column was kept at $0^{\circ} \mathrm{C}$ in an ice-water bath. Fractions were eluted in a linear salt gradient $(0-0.9 \mathrm{M} \mathrm{NaSCN}$ in $20 \mathrm{mM}$ pH $7.0 \mathrm{MES}$ buffer) with a $40 \mathrm{~mL}$ volume at a flow rate of $2.0 \mathrm{~mL} / \mathrm{min}$. Starting from 4 minutes of the elution time, fractions were collected in $0.5 \mathrm{~mL}$ aliquots every 15 seconds and the fractions were labeled again as the collection sequences (IEC1 through IEC40). By using a centrifugal filter (Millipore, $30000 \mathrm{MWCO}$ ), the fractions were exchanged with $\mathrm{D}_{2} \mathrm{O}$ for several times to remove the salts for optical measurements.

\section{(3) Characterization of FeRu SWNTs}

Uv-vis-NIR Spectroscopy. Uv-vis-NIR measurement of the FeRu grown SWNTs (and commercial Hipco and CoMoCAT) was carried out in $1 \mathrm{~mm}$ path-length quartz cell using Cary 6000i spectrophotometer with pure $\mathrm{H}_{2} \mathrm{O}$ as the background. The absorption spectrum of samples show distinct peaks in the $350 \mathrm{~nm}-1400 \mathrm{~nm}$ region, characteristic of highly dispersed individualized SWNTs. The IEC separated samples in $\mathrm{D}_{2} \mathrm{O}$ were housed in $1 \mathrm{~mm}$ path-length quartz cells and their absorption spectra were measured using a Varian Cary 5000 Uv-Vis-NIR spectrophotometer. $\mathrm{D}_{2} \mathrm{O}$ was used as a reference for background subtraction.

Photoluminescence Excitation/emission (PLE). Photoluminescence excitation/emission 
(PLE) measurements were performed utilizing a home-built setup. A short arc lamp (Osram XBO 75W/2 OFR 75W Xenon lamp installed into Oriel 66907 Arc Lamp Source) and a monochromator (Oriel 7400 Cornerstone 130 monochromator) were used to supply the excitation light in $550 \mathrm{~nm}-840 \mathrm{~nm}$ range in $10 \mathrm{~nm}$ steps. The excitation light was focused onto a sample placed in a $1 \mathrm{~mm}$ path quartz cuvette. The room temperature sample photoluminescence was collected at the opposite cuvette wall and the PL spectrum was recorded using a second monochromator (Acton SpectraPro 2300i) and a liquid Nitrogen cooled InGaAs array detector (Princeton Instruments OMA V 1024-2.2 $\mathrm{LN}$ ) in $900 \mathrm{~nm}-1450 \mathrm{~nm}$ range. The obtained PL spectra were scaled according to the measured excitation power (measured using Oriel 71580 calibrated Si photodiode) before obtaining PLE spectrum by interpolating the measured 30 PL spectra. The bandpass used for emission and excitation was $15 \mathrm{~nm}$.

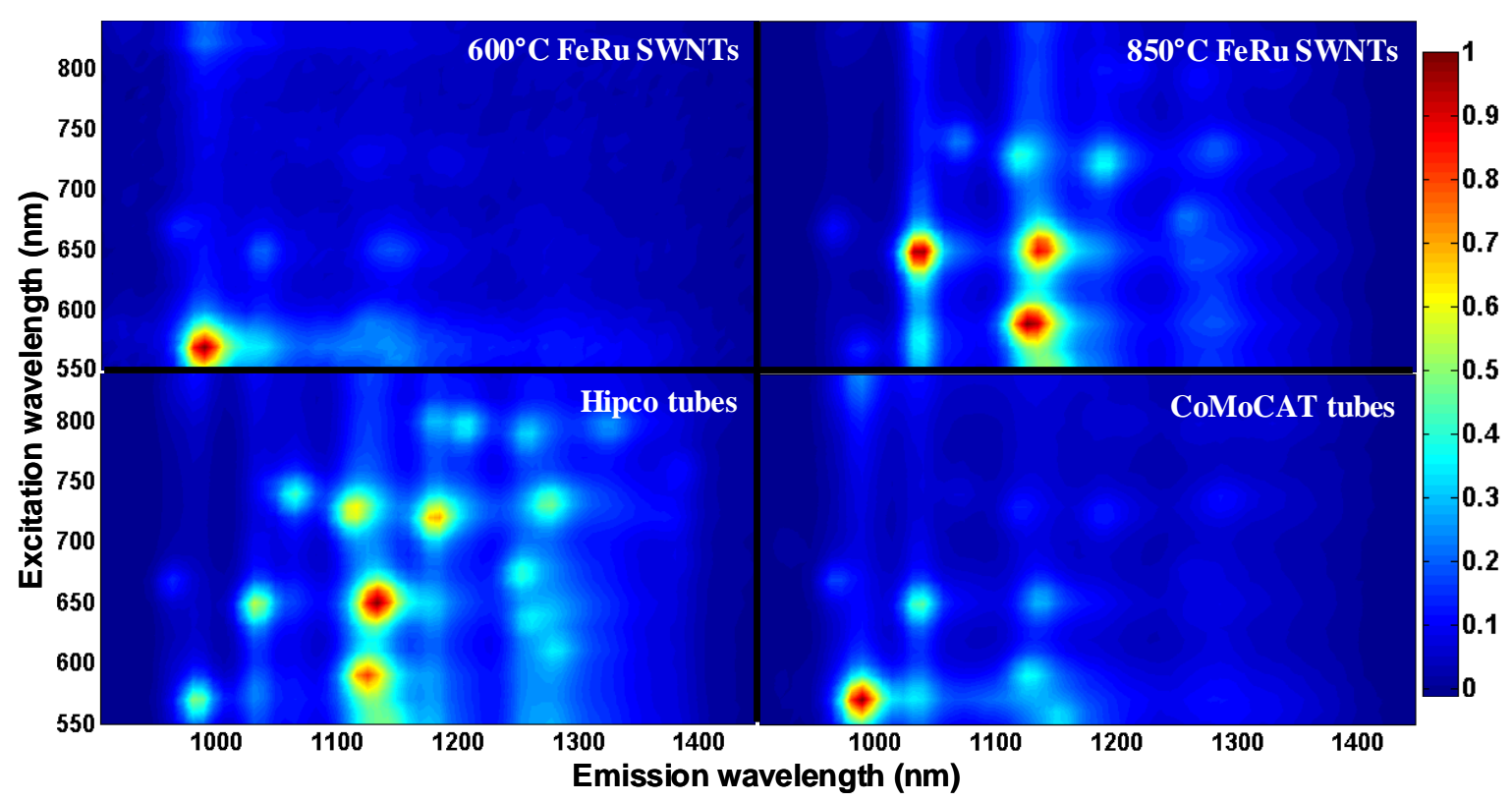

Fig. S1: PLE of FeRu, Hipco, and CoMoCAT tubes suspended in cholate. (a) $600^{\circ} \mathrm{C}$ 
growth $\mathrm{FeRu}$ tubes, (b) $850^{\circ} \mathrm{C}$ growth $\mathrm{FeRu}$ tubes, (c) Hipco tubes, (d) commercial CoMoCAT tubes. Note that we found that the SWNTs grown at $600^{\circ} \mathrm{C}$ appeared to be very difficult to debundle, likely due to strong interactions between the ultra-small diameter nanotubes in the bundles. This led to relatively dull UV-vis-NIR absorbance (see Fig. 1 in the main text) and PL peaks for the $(6,5)$ tube.

Atomic force microscopy (AFM). We deposited FeRu-grown SWNTs suspended in surfactant solutions onto silicon dioxide substrates for AFM imaging using a Nanoscope IIIa multimode instrument. The deposition was done by soaking a substrate into the SWNT suspension for 2 min, rinsing with isopropanol and blow-dry with Argon.
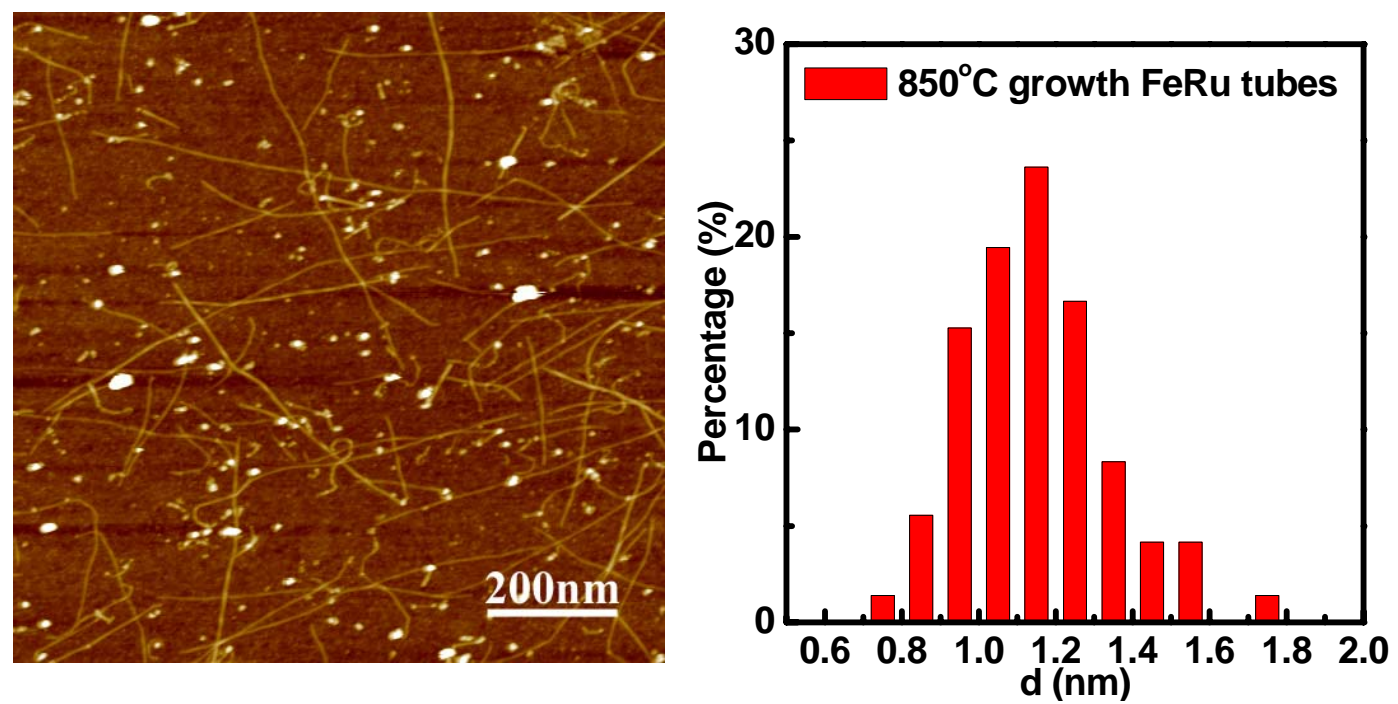

Fig. S2: Left: An AFM image of $850^{\circ} \mathrm{C}$ grown nanotubes from $\mathrm{FeRu}$ catalyst by methane CVD. Right: Diameter (topographic height from AFM data) histogram of nanotubes measured over 70 tubes. 